\title{
Proposed WfQ Based DyNamic BandWidTH Allocation For Mobile Wimax Application
}

\author{
Dr.B.Sridevi ${ }^{1}$, R.Anantha Priya ${ }^{2}$, V.S.Primiya ${ }^{3}$ \\ ${ }^{1}$ Assistant Professor, ${ }^{2,3}$ B.E.Student \\ Department of Electronics and Communication Engineering \\ Velammal College of Engineering and Technology, Madurai
}

\begin{abstract}
In this project, we proposed a framework to support heterogenous traffic with different QoS demand in WiMAX. This framework dynamically changes the bandwidth allocation (BA) for ongoing and new arrival connections based on network condition and service demand. The objective is to efficiently use the available bandwidth and provide QoS support in a fair manner. Dynamic allocation of spectrum prior to transmission can mitigate the starvation problem of Non Real time application. The WFQ based dynamic bandwidth allocation framework uses architecture that has packet scheduler scheme (PS), call admission policy and a dynamic bandwidth allocation mechanism. By the simulation result we have showed that this architecture could provide QoS support by being fair to all classes of services.
\end{abstract}

\section{KEYWORDS}

QoS in WiMAX, Dynamic Bandwidth Allocation, WFQ.

\section{INTRODUCTION}

Worldwide Interoperability for Microwave Access(WiMAX) will play an important role in the fixed broadband wireless access since it is more cost effective and faster to set up technology which is based on IEEE 802.16 standard that specifies the new air interface for microwave access, which was designed for wireless metropolitan area networks. WiMAX provides an affordable alternative for wireless broadband access supporting a variety of applications of different types including video conferencing, non-real-time large volume data transfer, traditional voice/data traffic throughput connection, and web browsing.In order to have the best controlled bandwidth allocation of the WiMAX network, IEEE 802.16e-2005 standard defines 5 types of service flow as following: UGS (Unsolicited Grant Service), rtPS(Real-Time Polling Service), ertPS (Extended Real-Time Polling Services), nrtPS (Non-Real Time PollingService) and BE (Best Effort). 


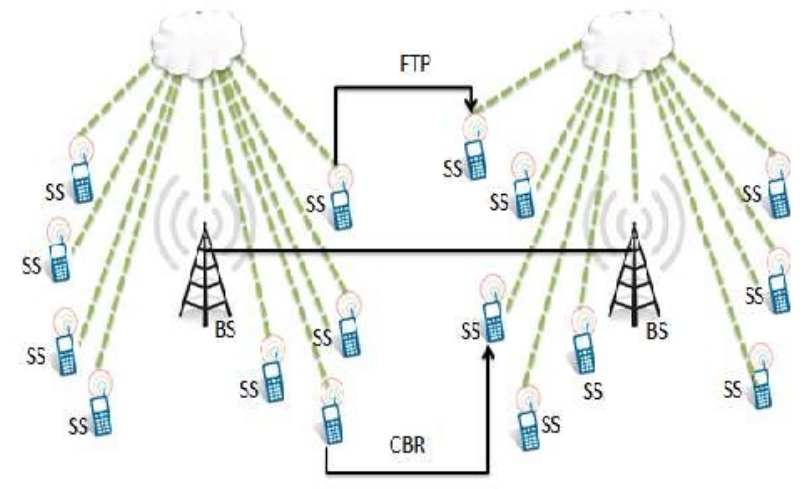

Fig1. WiMAX architecture

Each traffic flows requires different QoS to meet different QoS requirements of different traffic flows, an efficient queuing scheme is necessary due to the instability of wireless transmission environment therefore traffic scheduling is used to ensure that QoS requirements are met. Eventhough IEEE 802.16e was developed with QoS requirements, the scheduling standard does not specify traffic scheduling requirements, instead such implementation are render specific. Scheduling schemes are used to resolve conflict for shred resource in a network by allocating bandwidth to each requested users and determine the transmission priority.Each flow requires a certain minimum bandwidth to achieve its QoS. Bandwidth allocation to traffic classes should be in such a way that fairness criteria is met with. Hence, we propose a dynamic bandwidth allocation mechanism to achieve fair and efficient allocation.

Hence we propose a framework known as Dynamic QoS based Bandwidth Allocation. The aim is to resolve the problem of bandwidth allocation in IEEE802.16e.

There are several advantage in allocating the bandwidth dynamically: (1) all users are typically not connected to the network at one time (2) even when connected, users are not transmitting data at all times (3) most traffic occurs in bursts, there are gaps between packets of information that can be filled with other user traffic.

\section{RELATED WORKS}

The MAC scheduler in WiMAX technology is a crucial issue to design. The most important is to fulfill design constraints like QoSguarantee minimum throughput. Among several scheduling algorithms such as algorithms,Intra class scheduling (Round Robin, Weighted Round Robin, Deficit Round Robin,Delay Based), Inter class Scheduling (priority based algorithm, channel aware schedulers), the best suited WiMAX MAC scheduler is Weighted fair Queuing its dynamic nature make it more simple than other algorithms[1] .

The main objective of this work is to provide an implementation of IEEE 802.16 e standard using dynamic fuzzy based priority scheduler. Scheduling process is being done in two stages the requests are classified in terms of priority using fuzzy logic and the request are scheduled using neural networks[2]. 
International Journal of Computational Science and Information Technology (IJCSITY) Vol.2, No.2, May 2014

A detailed performance comparison of some scheduling algorithms such as Deficit Round Robin,Proportional Fair and Weighted Deficit Round Robin by taking into account their radio channels condition and the throughput improvement [3].

A scheduling scheme for the QoS classes in WiMAX, which considers the pricing factor in the optimization of resource usage and resource allocation.Wireless technologies are facing challenging issues with respect to new applications and increased user demands. Most of the challenging issues dealt with wireless signal propogation problem such as noise,fading,interference and so on. Although traditional problems such as the limited radio spectrum still remain among key problems, more sophisticated problems have evolved, which deal with QoS, resource allocation and resource reservation.One main issue in providing multimedia services: The proposed DQBA is implemented in three blocks, they are call admission control Tier1 and Tier2 .The admission control policy is implemented in the cache module. The packet scheduling is implemented in two levels, the first is intra class scheduling is done in tier 1 and interclass scheduling in tier 2.

Providing the required QoS level depends on four operations they are connection admission control, packet scheduling,traffic shaping and traffic policing. Packet scheduling consists of serving the resource requests which have been sent by the mobile users and queued at the base station level. Scheduling is based on two orthogonal components which are deciding the order of serving the user request and managing the service queue .Scheduling architecture for an IEEE 802.16 should guarantee efficient bandwidth utilization while providing fairness between users and responding correctly to the QoS constraints of real time application.

Two types of scheduling architecture are traditional scheduling architecture and new scheduling architecture. Traditional scheduling architecture are based on classical and simple scheduling algorithms which were used earlier in the wired technologies. Thus the traditional architectures use these algorithms initially while other architectures implement additional mechanism in order to extend these algorithms so that they become more suited to the IEEE 802.16 QoS context. The two modes allowed in the allocation of the bandwidth are : (i) Grant Per Connection(GPC) and (ii) Grant Per Subscriber Station (GPSS). When there are more connections its being compulsory for he users to use the $66 \mathrm{GHz}$ PHY specifications [5],[6] .

WiMAX Forum [10] according to IEEE 802.16e-2005standard defines the air interface of WiMAX system inPhysical (PHY) layer. WiMAX system can use bothOrthogonal Frequency Division Multiplexing (OFDM)and Orthogonal Frequency Division MultiplexingAccess (OFDMA) modulation technology, to effectivelyprovide multi-path access in Non-Line-ofSight(NLOS)environment. Thus, WiMAX will choose appropriatebandwidth at $1.25 \sim 20 \mathrm{MHz}$, according to the frequencyresource and service flow demand. WiMAX Physicallayer adopts to TDD mode, to choose proportion framefor Uplink and Downlink direction according to the

Unsolicited grant service (UGS) that supports constant bit rate such as voice application .Real Time Polling Service (rtPS) support real time data streams that contain variable size data packets which are issued as periodic intervals such as MPEG video.Extended Real Time Polling Service(ertPS) applicable with variable rate real time applications that require data rate with delay guarantees like VoIP with silence suppression.Non Real Time Polling Service (nrtPS) supports delaytolerance data streams that contains variable size data packets that require a minimum data rate like FTP. Best Effort (BE) support data streams that donot need any QoS guarantees like HTTP.While considering the pricing factor in case of resource optimization and allocation of 
International Journal of Computational Science and Information Technology (IJCSITY) Vol.2, No.2, May 2014

resources it provides a higher revenue for the service providers by the pricing model which is being provided by the Radio Resource Management (RRM) which was given by Belghith scheduling scheme. This clearly have an impact on the utilization overall performance operation. For various service classes different types of pricing schemes where introduced. In case of the economic impact the fairness in allocation of resources could be affected and can even make an impact

\begin{tabular}{|l|l|l|l|l|l|}
\hline SERVICE CLASS & CLASS 1 & CLASS 2 & CLASS 3 & CLASS 4 & CLASS 5 \\
\hline $\begin{array}{l}\text { TYPE OF } \\
\text { TRAFFIC }\end{array}$ & UGS & ertPS & rtPS & nrtPS & BE \\
\hline $\begin{array}{l}\text { PRIORITY } \\
\text { LEVEL }\end{array}$ & 5 & 4 & 3 & 2 & 1 \\
\hline $\begin{array}{l}\text { BANDWIDTH } \\
\text { REQUIREMENT }\end{array}$ & $5 \mathrm{Mbps}$ & $3 \mathrm{Mbps}$ & $1.5 \mathrm{Mbps}$ & $64 \mathrm{Kpbs}$ & 32 Kpbs \\
\hline APPLICATION & Voice & video & Video & Audio & Email \\
\hline
\end{tabular}

Table1.Types Of Traffic And Application

\section{PROPOSED WORK}

The objective of this framework is to provide the best Quality of Service and which would pave way for the reduction in the number of call drops, even the starvation problem and wastage of resources in the fixed bandwidth allocation can be solved. This can be given by the methodologies which follows Call Admission Control followed by Tier1 and then Tier2. For this task the operations performed are scheduling, bandwidth allocation and admission control. The five different classes follows Unsolicited Grant Service(UGS), Extended Real Time Polling Service(ertPS), Non Real Time Polling Service(nrtPS), Real Time Polling Service(rtPS) and Best Effort(BE).

\subsection{Packet Scheduling}

In case of UGS it uses Earliest Deadline First (EDF) which includes the latency period and the arrival timeit is also a kind of priority based algorithm which provides the delay guarantee service for its users. In case of rtPS and ertPS, Weighted Fair Queuing (WFQ) is being used. It result in the superior performance compared with the other algorithms with the presence of the variable sized packets and the reason behind that is, it does not consider the starting time of the packets. The nrtPS uses Round Robin(RR) algorithm. It is the most basic scheduling algorithm it can distribute the channel resources to all the substation. In tier2, Weighted Fair Queuing can ensure satisfactory response time to all kinds of critical applications, such as interactive and transaction based ones, that are being intolerant to performance degradation.A Dynamic WFQ [9][10] is able to dynamically and consistently adapt the queue weights according to the time-variant amount of the incoming traffic and the preassigned target QoS. 


\section{STEP 1:}

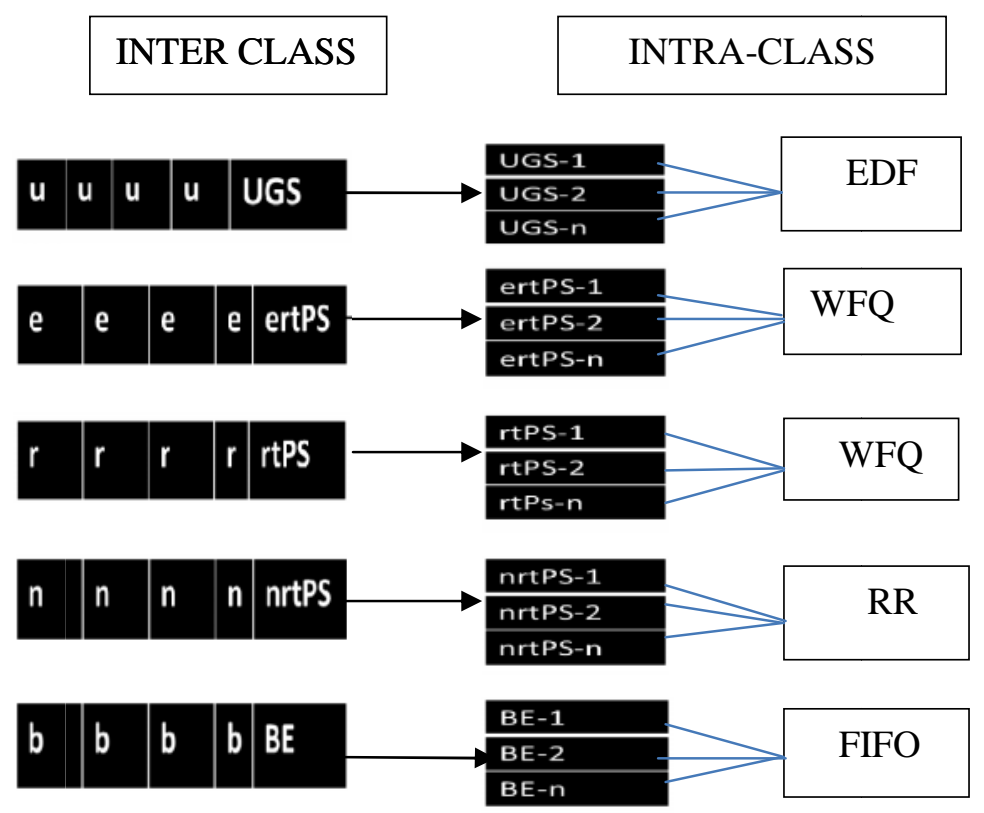

Fig 2. Intra class scheduling

\subsection{Bandwidth Allocation}

The bandwidth is dynamically allocated based on BVal.BVal is calculated by considering fairness, utilization and delay bound. BVal is an important parameter used for packet scheduling, call admission control and dynamic bandwidth allocation by integrating traffic and network characteristics

\section{Algorithm 1 :BVal calculation}

1: GET incoming packets

2: GETutilization,fairness and delay bound for each service request.

3:GET the requested bandwidth

4: For each service request

5: if $\left(\mathrm{F}^{(\mathrm{i}, \mathrm{j})} \geq \mathrm{F}_{(\mathrm{i}, \mathrm{i}-1)}{ }^{(\mathrm{i}, \mathrm{j})}\right)$ and $\left(\mathrm{U}^{(\mathrm{i}, \mathrm{j})} \geq \mathrm{U}_{(\mathrm{i}, \mathrm{j}-1)}\right)$

6: then $\left(\mathrm{BVal}^{(\mathrm{i}, \mathrm{j}+1)}=\mathfrak{£}^{(\mathrm{i}, \mathrm{j}, \mathrm{j})}\right)$

7: $\operatorname{elseif}\left(\mathrm{F}^{(\mathrm{i}, \mathrm{j})}<\mathrm{F}_{(\mathrm{i}, \mathrm{i}-\mathrm{i})}(\mathrm{i}, \mathrm{j})\right.$

8: then $\left(\mathrm{BVal}^{(\mathrm{i}, \mathrm{j}+1)}\right)=\mathfrak{f}_{(\mathrm{i}, \mathrm{j}-1)}^{(\mathrm{i}, \mathrm{j})}\left(1+\mathrm{F}_{(\mathrm{i}, \mathrm{j}-1)}\right)$

9: $\operatorname{elseif}\left(\mathrm{U}^{(\mathrm{i}, \mathrm{j})}<\mathrm{U}_{(\mathrm{i}, \mathrm{i}-1)}(\mathrm{i}, \mathrm{j}) \mathrm{(}\right)$

10: then $\left(\mathrm{BVal}^{(\mathrm{i}, \mathrm{j}+1)}=£_{(\mathrm{i}, \mathrm{j}-1)}^{(\mathrm{i}, \mathrm{j})}\left(1+\mathrm{U}_{(\mathrm{i}, \mathrm{j}-1)}\right)\right.$

11:elseif $\left(F^{(i, j)}<F_{(i, j-1)}\right)$

12: then $\left(B \mathrm{Bal}^{(\mathrm{i}, \mathrm{j}+1)}=\mathfrak{£}^{(\mathrm{i}, \mathrm{j})}\left(1+\mathrm{F}^{(\mathrm{i}, \mathrm{j})}+\mathrm{U}^{(\mathrm{i}, \mathrm{j})}\right)\right.$

13:if $(\mathrm{D}<10)$

14: thenbdcap $=\mathrm{BVal} * 15$

15:if $(\mathrm{D}<20)$ 
International Journal of Computational Science and Information Technology (IJCSITY) Vol.2, No.2, May 2014 16: thenbdcap $=\mathrm{BVal} * 10$

$17:$ if $(\mathrm{D}<30)$

18:thenbdcap $=\mathrm{BVal} * 5$

19: if $(\mathrm{D}<40)$

20: thenbdcap $=\mathrm{BVal} * 2.5$

13: Endfor

14: ReturnBVal value

We define fairness as the ratio of the allocated bandwidth by the requested bandwidth,

$$
F^{(i, j)}=\left(\operatorname{Abnw}^{(i, j)} / \operatorname{Rbnw}^{(i, j)}\right)
$$

And utilization is defined as the ratio of the used network resources to the available resources

$$
\mathrm{U}^{(\mathrm{i}, \mathrm{j})}=\left(\mathrm{T}^{(\mathrm{i}, \mathrm{j})} / \operatorname{Abnw}^{(\mathrm{i}, \mathrm{j})}\right)
$$

Residual bandwidth= allocated bandwidth - requested bandwidth

Bandwidth is allocated dynamically based on BVal.

\section{STEP 2 :}

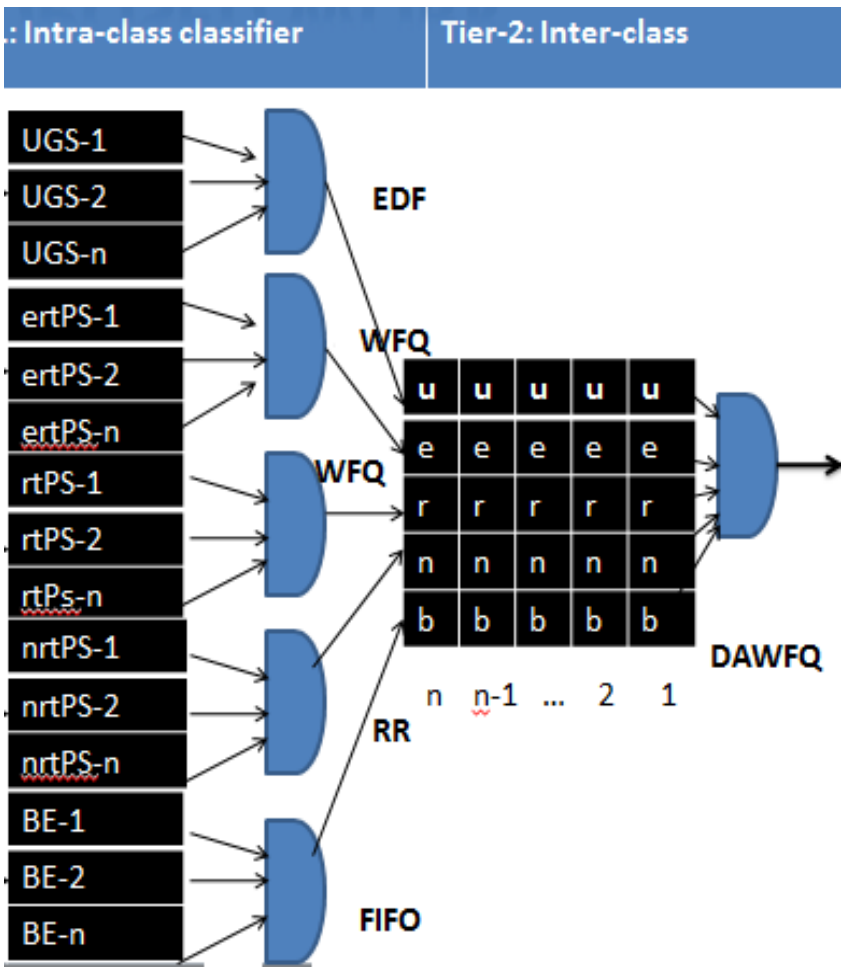

Fig 3. Inter class scheduling. 
International Journal of Computational Science and Information Technology (IJCSITY) Vol.2, No.2, May 2014

\subsection{CAC (Call Admission Control)}

Usually CAC procedure is being implemented at the BS that ensures the load supplied through the subscriber station (SS) can be taken over by a network. The choice of the call admission policy that is to be adopted in an IEEE802.16 network is being strongly associated with the scheduling mechanisms used. CAC restricts the access to the network to prevent congestion or service degradation for the users those are already being accepted. It also prevents the system before being overloaded.

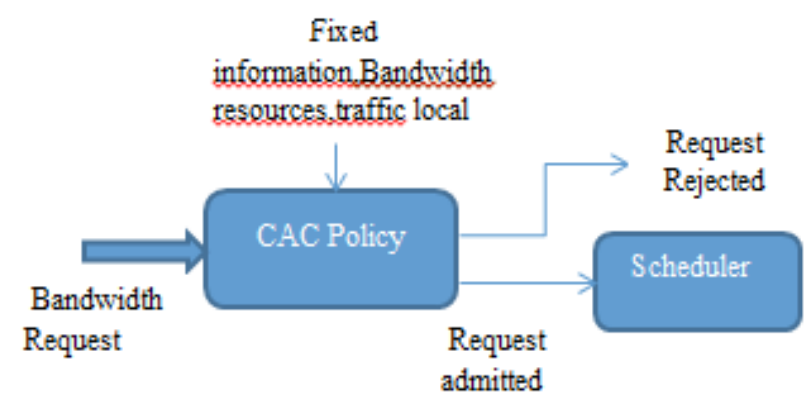

Fig 3.Call Admission Control

\section{SIMULATION AND RESULTS}

This section discusses the performance evaluation of the proposed algorithm. The simulations were conducted using MATlab.

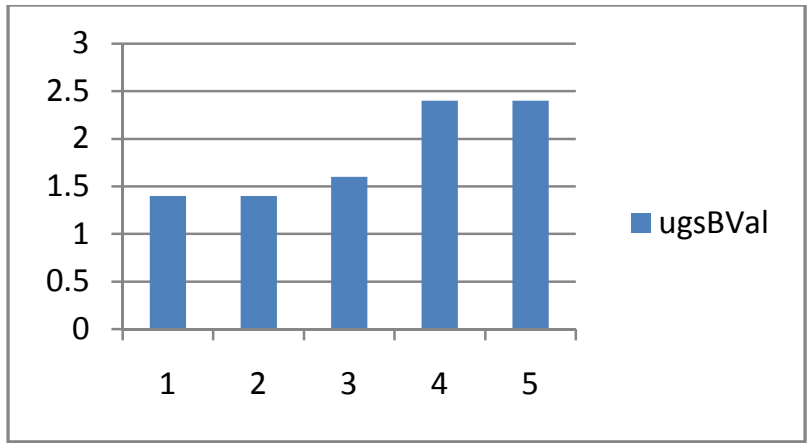

Fig 4: Intra class scheduling of UGS users 
International Journal of Computational Science and Information Technology (IJCSITY) Vol.2, No.2, May 2014

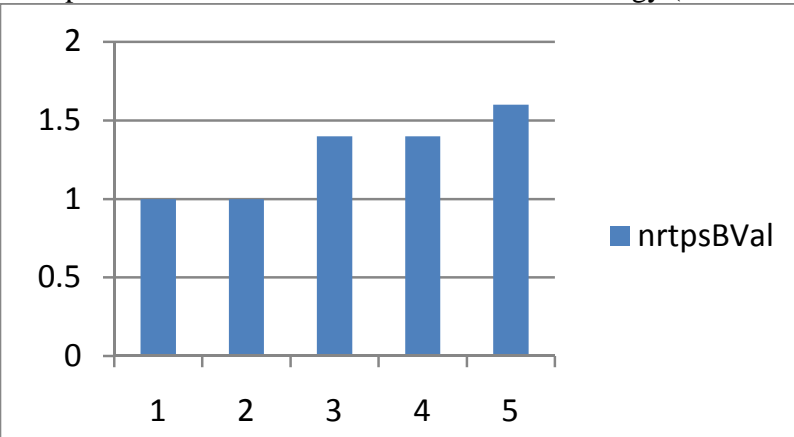

Fig 5 .Intra class scheduling of nrtPS users

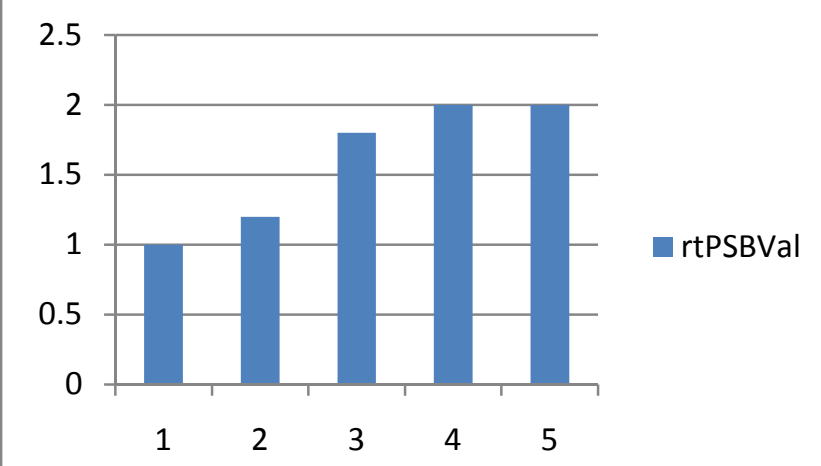

Fig 5.Intra class scheduling of rtPS users

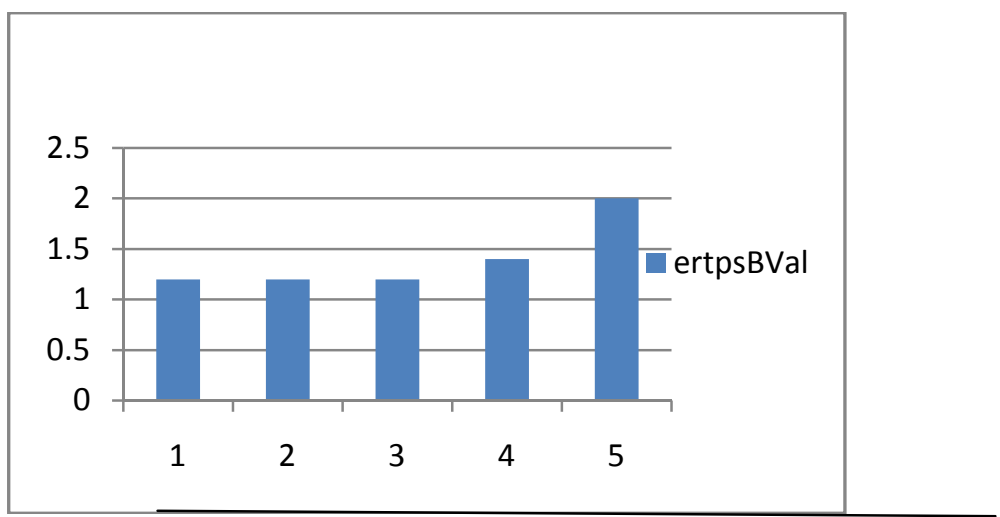

Fig 6. Intra class scheduling of ertPS users 
International Journal of Computational Science and Information Technology (IJCSITY) Vol.2, No.2, May 2014

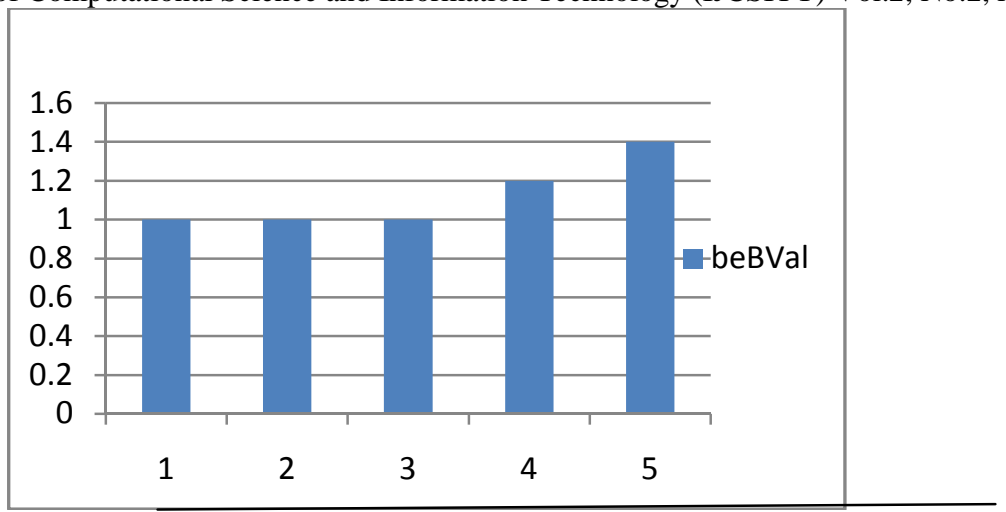

Fig 7. Intra class scheduling of BE users

This result shows that intra class scheduling is done among all the five types of service classes by comparing the corresponding the utilisation and fairness of each packets .Fig 4 shows that UGS packets are ordered within the UGS class itself i.e.intraclass scheduling. Likewise nrtPS,rtPS,ertPS and BE traffic is ordered which is shown in Fig 5, 6, 7 respectively

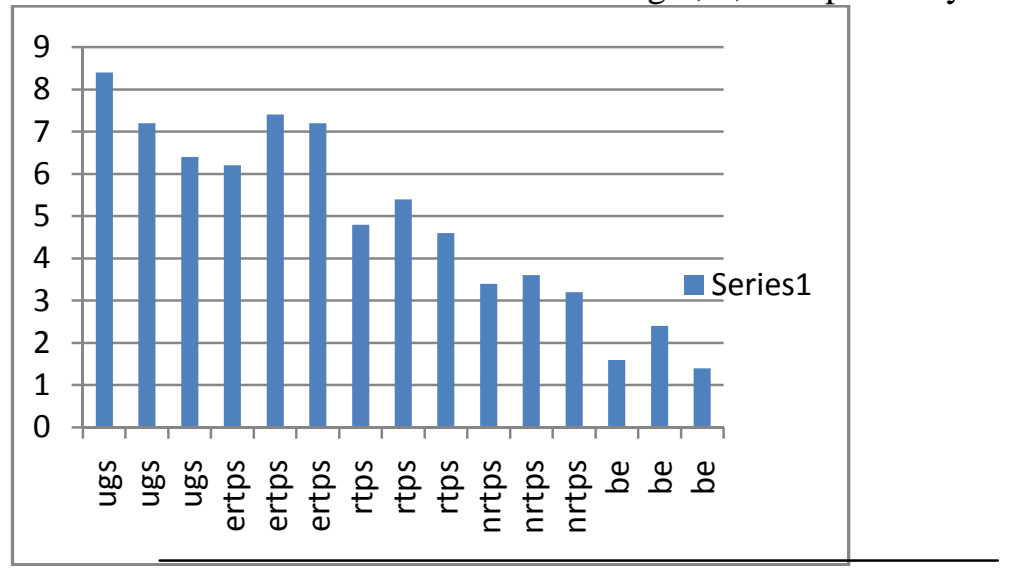

Fig 8. Fixed bandwidth allocation

We can infer from the fig. 8 that only higher priority is getting the service leaving behind the lower priorities, thus higher priority starve the bandwidth of lower priority. This cause the starvation problem. 
International Journal of Computational Science and Information Technology (IJCSITY) Vol.2, No.2, May 2014

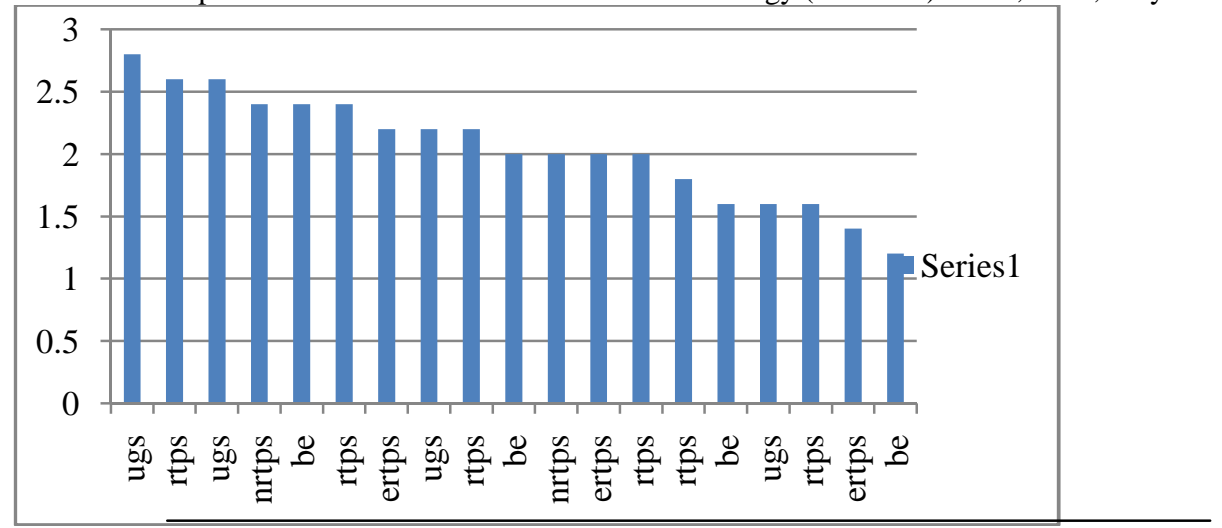

Fig 9 : Inter class scheduling

A traffic seperation in both inter and intra class levels is shown and the QoS suport is being guaranteed by both real time and non real time application. Thus if the bandwidth available is less than the requested by the higher priority, then the lower priority will be served in order to provide a fair solution for the lower priorities. Fig 9 shows that lower priority applications are not completely suffering from starvation problem.

\section{CONCLUSION}

In this paper we proposed a framework which supports all kinds of service flows and provide a fair share of bandwidth by using several strategies such as multilevel queuing discipline, QoS differentiation and unused bandwidth reallocation, thus the network performence is being improved .This result indicate that eventhough the low priority flows or not completely starved, the higher priority applications are consuming a considerable portion of the bandwidth thus the simulatuion result shows that the the utilization has been increased which directly affects the reduction of the call blocking property.

\section{REFERENCE}

[1] Comparitive study of different scheduling algorithms for WiMAX MAC scheduler design.Gajanan M. Galshetwar, Prof. AmuthaJayakumar, Yashika Mittal

[2] An Enhanced Scheduling Scheme for QoS in mobile WiMAX networks. D.DavidNeelaPon Kumar, Jithin Kumar, K. Arun Kumar, K.Murugesan

[3] Comparison Of Different Scheduling Algorithms For WiMAX base station. JaniLakkakori, AlexandarSayenko, JaniMoilanen. Las Vegas, Nevada: IEEE Wireless Communication and Networking Conference WCNC, 2008, Vol. 08. 1525-3511.

[4] A.Belghith, "Pricing Based Schedulers for WiMAX ", in Proc. IEEE Int Conf. Wireless Mobile Computer, Netw. Communication WIMOB , Oct 12-14, 2009, pp.202-207

[5] IEEE 802.16 Standard (2004), "IEEE Standard For Local and Metropolitan Area Networks - Part 16: Air Interface for Fixed Broadband Wireless Access Systems".

[6] Robert Holte, Al Mok, Louis Rosier, Igor Tulcjinsky and Donald Varvel, "The Pinwheel: A Real Time Scheduling Problem". Proc of the 22nd Hawaii International Conference on System Science, 1989, pp 693-702.

[7] K .Wongthavarawat and A.Ganz, " Packet Scheduling for QoS Support in IEEE802.16 broadband wireless access systems,” Int. J. Commun. Syst., vol. 16, no. 1,pp. 81-96, Feb.2003. 
International Journal of Computational Science and Information Technology (IJCSITY) Vol.2, No.2, May 2014

[8] J.Chen, W.Jiao, and Q.Guo, "Providing integrated QoS control for IEEE 802.16 broadband wireless access systems ," in Proc. VTC,Dallas,TX,2005, vol. 2, pp. 1254-1258,

[9] Chin-Chang Li, Shiano-Li Tsao, Mengchengchen, Yaeli Sun, Yueh-Min Huang, Proportional Delay Differentation Service Based on Weighted Fair Queuing.

[10] Kun Pang Xiaokang Lin JunliXuedaoGu Nat, Dynamic WFQ scheduling for real-time traffic in wireless ATM links, CommunicationTechnology Proceedings, 2000

\section{Authors}

B.Sridevi, Assistant Professor of ECE Department of Velammal College of Engineering \& Technology, Madurai, obtained her B.E., degree from A.C.C.E.T Karaikudi, Madurai Kamaraj University, Madurai and M.E. degree from Anna University, Chennai. She has 2 years of Industrial experience, 10 years of Teaching,and Research experience. Pursuing Ph.D. in Anna University, Tirunelveli in Networking. She published many research papers in International journals, national and international conferences. Her area of research includes Network Security, Wireless Networks.

V. S. Primiya, pursuing Bachelor of engineering in Velammal College of Engineering and Technology, Madurai-625009. Her area of interest is wireless networks
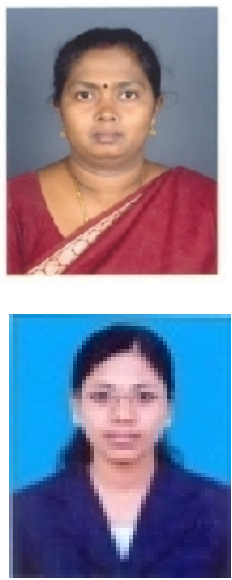

R.AnanthaPriya, pursuing Bachelor of engineering in Velammal College of Engineering and Technology, Madurai-625009. Her area of interest is wireless networks

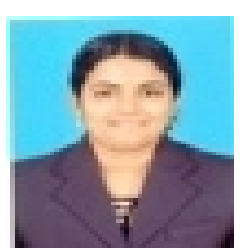

